FACTA UNIVERSITATIS

Series: Mechanical Engineering Vol. 16, N ${ }^{\circ} 1,2018$, pp. 9 - 18

https://doi.org/10.22190/FUME171121003W

Original scientific paper

\title{
DUGDALE-MAUGIS ADHESIVE NORMAL CONTACT OF AXISYMMETRIC POWER-LAW GRADED ELASTIC BODIES
}

UDC 539.3

\section{Emanuel Willert}

\author{
Berlin University of Technology, Berlin, Germany
}

\begin{abstract}
A closed-form general analytic solution is presented for the adhesive normal contact of convex axisymmetric power-law graded elastic bodies using a DugdaleMaugis model for the adhesive stress. The case of spherical contacting bodies is studied in detail. The known JKR- and DMT-limits can be derived from the general solution, whereas the transition between both can be captured introducing a generalized Tabor parameter depending on the material grading. The influence of the Tabor parameter and the material grading is studied.
\end{abstract}

Key Words: Adhesive Contact, Power-Law Elastic Grading, Dugdale-Maugis Model, Axial Symmetry, Method of Dimensionality Reduction

\section{INTRODUCTION}

Propelled by the technological demand for versatile high-performance materials and the study of biological materials and contact solutions, living nature developed in several circumstances, Functionally Graded Materials (FGM), i.e. media with continuously inhomogeneous mechanical properties, have encountered a lot of scientific interest and research in the past years. The use of FGM is proven to be possibly beneficial in physical [1] and biological [2] applications. Whereas rigorous solutions for non-adhesive contact problems of FGM, at least for some special forms of inhomogeneity, have been available for quite a long time [3-5], the adhesive contact of FGM is still in the focus of current research [6-9]. These latter studies, nonetheless, only concern the limiting case of a negligible range of the adhesive interaction, established by Johnson, Kendall and Roberts (JKR, [10]) in 1971. After Derjaguin, Muller and Toporov (DMT, [11]) a few years later presented a different theory of long-range adhesive interactions giving a different result

Received November 21, 2017 / Accepted January 11, 2018

Corresponding author: Emanuel Willert

Technische Universität Berlin, Sekr. C8-4, Straße des 17. Juni 135, D-10623 Berlin

E-mail: e.willert@tu-berlin.de 
for the critical pull-off force in a parabolic contact, a discussion started, which was only finally resolved by Maugis [12], who - based on a model of the adhesive stress first introduced by Dugdale [13] - was able to show the transition between what was proven by Tabor [14] to be correct descriptions of limiting cases. The present paper generalizes Maugis' solution for the adhesive contact of homogeneous spheres to arbitrary axisymmetric bodies with elastic-grading in form of a power-law. As the contact problem of interest can be ascribed to the frictionless, non-adhesive normal contact of power-law graded elastic materials a solution procedure based on the Method of Dimensionality Reduction (MDR) can be applied.

\section{GENERAL AXISYMMETRIC SOLUTION}

We consider elastic grading of the Young modulus $E$ with depth $z$ in form of a powerlaw:

$$
E(z)=E_{0}\left(\frac{z}{z_{0}}\right)^{k}, \quad-1<k<1 .
$$

Thereby constants $E_{0}$ and $z_{0}$ as well as Poisson ratio $v$ may be different for the contacting bodies. Exponent $k$, however, needs to be the same for both of them. As the exponent may take positive or negative values, both soft surfaces with a hard core and hard surfaces with a soft core can be studied.

It has been shown that the frictionless normal contact of axisymmetric power-law graded elastic bodies can be exactly mapped onto a plain contact of a rigid profile $g$ with a one-dimensional foundation of independent linear springs, each in distant $\Delta x$ from each other $[15,16]$. Thereby the equivalent plain profile $g=g(x)$ within this mapping procedure called Method of Dimensionality Reduction (MDR) can be calculated from the axisymmetric gap $f=f(r)$ between the non-deformed three-dimensional bodies by the integral transform:

$$
g(x)=|x|^{1-k} \int_{0}^{|x|} \frac{f^{\prime}(r)}{\sqrt{\left(x^{2}-r^{2}\right)^{1-k}}} \mathrm{~d} r .
$$

Stiffness $\Delta k_{z}$ of a single spring at position $x$ is given by the expression:

$$
\Delta k_{z}(x)=c_{N}\left(\frac{|x|}{z_{0}}\right)^{k} \Delta x,
$$

with:

$$
c_{N}:=\left(\frac{1-v_{1}^{2}}{H\left(k, v_{1}\right) E_{01}}+\frac{1-v_{2}^{2}}{H\left(k, v_{2}\right) E_{02}}\right)^{-1} .
$$

Dimensionless auxiliary function $H$ can be determined from exponent $k$ and Poisson's ratio according to: 


$$
H(k, v):=\frac{2(1+k) \cos (k \pi / 2) \Gamma(1+k / 2)}{\sqrt{\pi} C(k, v) \beta(k, v) \sin [\beta(k, v) \pi / 2] \Gamma[(1+k) / 2]},
$$

with:

$$
C(k, v):=\frac{2^{1+k} \Gamma[(3+k+\beta(k, v)) / 2] \Gamma[(3+k-\beta(k, v)) / 2]}{\pi \Gamma(2+k)}
$$

and

$$
\beta(k, v):=\sqrt{(1+k)\left(1-\frac{k v}{1-v}\right)}
$$

and Gamma function $\Gamma$.

Note that the spatial distribution of the spring stiffness in Eq. (3) obeys the same power-law as the elastic grading. If equivalent profile $g$ is pressed into the foundation of springs by an indentation depth $d$ the vertical spring displacement $w_{1 \mathrm{D}}(x)$ in the area of direct contact is elementarily given by:

$$
w_{1 \mathrm{D}}(x)=d-g(x), \quad|x| \leq a,
$$

with contact radius $a$. Normal force $F_{N}$ as well as the local distributions of pressure $p$ and relative displacement $w$ in the original three-dimensional system can be calculated from $w_{1 \mathrm{D}}(x)$ according to:

$$
\begin{aligned}
F_{N} & =\frac{c_{N}}{z_{0}^{k}} \int_{-\infty}^{\infty} w_{1 \mathrm{D}}(x)|x|^{k} \mathrm{~d} x, \\
p^{*}(r) & =-\frac{c_{N}}{\pi z_{0}^{k}} \int_{r}^{\infty} \frac{w_{1 \mathrm{D}}^{\prime}(x) \mathrm{d} x}{\sqrt{\left(x^{2}-r^{2}\right)^{1-k}}}, \\
w(r) & =\frac{2 \cos (k \pi / 2)}{\pi} \int_{0}^{r} \frac{x^{k} w_{1 \mathrm{D}}(x) \mathrm{d} x}{\sqrt{\left(r^{2}-x^{2}\right)^{1+k}}} .
\end{aligned}
$$

The second of these latter Eqs. (9) can be inverted to give:

$$
w_{1 \mathrm{D}}(x)=\frac{2 z_{0}^{k} \cos (k \pi / 2)}{c_{N}} \int_{x}^{\infty} \frac{r p(r) \mathrm{d} r}{\sqrt{\left(r^{2}-x^{2}\right)^{1+k}}} .
$$

If we now assume a Dugdale model of a constant adhesive stress $\sigma_{0}$ within the adhesive zone with radius $b$ :

$$
p_{\text {adh }}(r)=-\sigma_{0}, \quad r \leq b,
$$

the corresponding displacements in the MDR model are due to Eq. (10) given by:

$$
w_{1 \mathrm{D}, \mathrm{adh}}(x)=-\frac{2 z_{0}^{k} \cos (k \pi / 2) \sigma_{0}}{c_{N}(1-k)} \sqrt{\left(b^{2}-x^{2}\right)^{1-k}}, \quad|x| \leq b .
$$

Hence, the one-dimensional displacements in the Dugdale-Maugis adhesive contact are: 


$$
w_{1 \mathrm{D}}(x)=\left\{\begin{array}{lc}
d-g(x), & |x| \leq a, \\
-\frac{2 z_{0}^{k} \cos (k \pi / 2) \sigma_{0}}{c_{N}(1-k)} \sqrt{\left(b^{2}-x^{2}\right)^{1-k}}, & a<|x| \leq b .
\end{array}\right.
$$

For the three-dimensional stresses to be finite at the edge of direct contact these displacements must be continuous at $x=a$, which results in:

$$
d=g(a)-\frac{2 z_{0}^{k} \cos (k \pi / 2) \sigma_{0}}{c_{N}(1-k)} \sqrt{\left(b^{2}-a^{2}\right)^{1-k}} .
$$

The total external normal force is due to the first of Eqs. (9) given by:

$$
\begin{aligned}
F_{N} & =\frac{2 c_{N}}{z_{0}^{k}} \int_{0}^{a}[d-g(x)] x^{k} \mathrm{~d} x-F_{\text {adh }}, \\
F_{\text {adh }} & :=\sigma_{0} b^{2}\left[\pi-\frac{4 \cos (k \pi / 2)}{1-k^{2}}\left(\frac{a}{b}\right)^{1+k}{ }_{2} \mathrm{~F}_{1}\left(\frac{k-1}{2}, \frac{1+k}{2} ; \frac{3+k}{2} ; \frac{a^{2}}{b^{2}}\right)\right],
\end{aligned}
$$

with the hypergeometric function:

$$
\begin{aligned}
{ }_{2} \mathrm{~F}_{1}(a, b ; c ; z) & :=\sum_{n=0}^{\infty} \frac{(a)_{n}(b)_{n}}{(c)_{n}} \frac{z^{n}}{n !}, \quad|z|<1, \\
(x)_{n} & :=\frac{\Gamma(x+n)}{\Gamma(x)} .
\end{aligned}
$$

Radius $b$ of the adhesive zone is not known a priori but can be determined from the condition that the gap between the deformed surfaces at $r=b$ has to equal the range $h$ of the adhesive stresses. As the gap between the deformed surfaces can be easily calculated from three-dimensional relative displacement $w$, indentation depth $d$ and axisymmetric non-deformed gap $f$, we obtain the additional relation

$$
h=w(r=b)-d+f(r=b) .
$$

to close the equation system. Evaluating Eq. (17) with the help of the third of Eqs. (9) and using the identity:

$$
\frac{2 \cos (k \pi / 2)}{\pi} \int_{0}^{b} \frac{x^{k}[d-g(x)] \mathrm{d} x}{\sqrt{\left(b^{2}-x^{2}\right)^{1+k}}}=d-f(b)
$$

one obtains:

$$
\begin{aligned}
& h=-\frac{2 \cos (k \pi / 2)}{\pi} \int_{a}^{b} \frac{x^{k}[d-g(x)] \mathrm{d} x}{\sqrt{\left(b^{2}-x^{2}\right)^{1+k}}}- \\
& -\frac{4}{\pi} \frac{\cos ^{2}\left(\frac{k \pi}{2}\right)}{1-k^{2}} \frac{\sigma_{0} z_{0}^{k} b^{1-k}}{c_{N}}\left[\frac{\Gamma(1-k) \Gamma\left(\frac{3+k}{2}\right)}{\Gamma\left(\frac{3-k}{2}\right)}-\left(\frac{a}{b}\right)^{1+k}{ }_{2} \mathrm{~F}_{1}\left(k, \frac{1+k}{2} ; \frac{3+k}{2} ; \frac{a^{2}}{b^{2}}\right)\right] .
\end{aligned}
$$


Equations (14), (15) and (19) completely solve the given contact problem. In the homogeneous case $k=0$ they are reduced to the axisymmetric generalization of Maugis' results given very recently by Popov et al. [17]. The stresses in the area of direct contact could theoretically be calculated inserting Eq. (13) into the second of Eqs. (9).

\section{THE JKR LIMIT}

It is of course possible to retrieve the known solution in the JKR limit of adhesion from the relations derived in the previous section. For this purpose we study the limit of negligible adhesion range $h \rightarrow 0$, whereas the surface energy per unit area, $\Delta \gamma=\sigma_{0} h$, is kept constant. In this case the radius of the adhesive zone can be written in the form:

$$
b=a(1+\varepsilon),
$$

with a small parameter $\varepsilon$. Using the linearization:

$$
g(a+\Delta x) \approx g(a)+g^{\prime}(a) \Delta x,
$$

performing the integration and neglecting all terms of higher than first order in $\varepsilon$ leads to:

$$
\begin{aligned}
& d \approx g(a)-\frac{2 \cos (k \pi / 2)}{1-k} \frac{\sigma_{0} z_{0}^{k} a^{1-k}}{c_{N}} \sqrt{(2 \varepsilon)^{1-k}}, \\
& h \approx-\frac{2}{\pi} \cos \left(\frac{k \pi}{2}\right)\left[\frac{d-g(a)}{1-k} \sqrt{(2 \varepsilon)^{1-k}}\right]-\frac{2}{\pi} \frac{\cos ^{2}(k \pi / 2)}{(1-k)^{2}} \frac{\sigma_{0} z_{0}^{k} a^{1-k}}{c_{N}}(2 \varepsilon)^{1-k} .
\end{aligned}
$$

Hence,

$$
h=\frac{\Delta \gamma}{\sigma_{0}} \approx \frac{2}{\pi} \frac{\cos ^{2}(k \pi / 2)}{(1-k)^{2}} \frac{\sigma_{0} z_{0}^{k} a^{1-k}}{c_{N}}(2 \varepsilon)^{1-k}
$$

and therefore:

$$
d \approx g(a)-\sqrt{\frac{2 \pi \Delta \gamma z_{0}^{k}}{c_{N}} a^{1-k}},
$$

which perfectly coincides with the known solution in the JKR limit [8]. The normal force via the same mechanism is also reduced to the known relation:

$$
F_{N} \approx \frac{2 c_{N}}{z_{0}^{k}} \int_{0}^{a}[d-g(x)] x^{k} \mathrm{~d} x
$$

Note that Eq. (23) is actually independent of the profiles of the contacting bodies.

\section{PARABOlic CONTACT}

Let us now consider the specific case of parabolic contact with the radius of curvature $R$, i.e.:

$$
f(r)=\frac{r^{2}}{2 R}
$$


The equivalent profile is accordingly:

$$
g(x)=\frac{x^{2}}{R(1+k)} .
$$

Thus, evaluating the general solution derived above, the solution of the Dugdale-Maugis adhesive normal contact problem in case of power-law elastic grading is given by:

$$
\begin{gathered}
d=\frac{a^{2}}{R(1+k)}-\frac{2 z_{0}^{k} \cos (k \pi / 2) \sigma_{0}}{c_{N}(1-k)} \sqrt{\left(b^{2}-a^{2}\right)^{1-k}}, \\
F_{N}=\frac{4 c_{N}}{R z_{0}^{k}} \frac{a^{3+k}}{(1+k)^{2}(3+k)}-\sigma_{0} b^{2}\left[\pi+\frac{4 \cos (k \pi / 2)}{1-k^{2}}\left(\frac{a}{b}\right)^{1+k} .\right. \\
\left.\cdot\left\{\sqrt{\left(1-\frac{a^{2}}{b^{2}}\right)^{1-k}}-{ }_{2} \mathrm{~F}_{1}\left(\frac{k-1}{2}, \frac{1+k}{2} ; \frac{3+k}{2} ; \frac{a^{2}}{b^{2}}\right)\right\}\right] .
\end{gathered}
$$

Radius $b$ of the adhesive zone can be determined from the condition:

$$
\begin{aligned}
h & =d\left[\frac{2}{\pi} \frac{\cos (k \pi / 2)}{1+k}\left(\frac{a}{b}\right)^{1+k}{ }_{2} \mathrm{~F}_{1}\left(\frac{1+k}{2}, \frac{1+k}{2} ; \frac{3+k}{2} ; \frac{a^{2}}{b^{2}}\right)-1\right]- \\
& -\frac{b^{2}}{2 R}\left[\frac{4}{\pi} \frac{\cos (k \pi / 2)}{(1+k)(3+k)}\left(\frac{a}{b}\right)^{3+k}{ }_{2} \mathrm{~F}_{1}\left(\frac{1+k}{2}, \frac{3+k}{2} ; \frac{5+k}{2} ; \frac{a^{2}}{b^{2}}\right)-1\right]- \\
- & \frac{4}{\pi} \frac{\cos ^{2}\left(\frac{k \pi}{2}\right)}{1-k^{2}} \frac{\sigma_{0} z_{0}^{k} b^{1-k}}{c_{N}}\left[\frac{\Gamma(1-k) \Gamma\left(\frac{3+k}{2}\right)}{\Gamma\left(\frac{3-k}{2}\right)}-\left(\frac{a}{b}\right)^{1+k}{ }_{2} \mathrm{~F}_{1}\left(k, \frac{1+k}{2} ; \frac{3+k}{2} ; \frac{a^{2}}{b^{2}}\right)\right] .
\end{aligned}
$$

Introducing the normalized variables

$$
\tilde{d}:=\frac{d}{\left|d_{c}\right|}, \quad \tilde{a}:=\frac{a}{a_{c}}, \quad \tilde{F}_{c}:=\frac{F_{N}}{\left|F_{c}\right|}, \quad m:=\frac{b}{a},
$$

with the critical values in the JKR limit under force-controlled boundary conditions [6]:

$$
\begin{aligned}
& d_{c}=\frac{k-1}{(1+k)(3+k) R}\left[\frac{\pi(1+k)^{2}(3+k)^{2} R^{2} \Delta \gamma z_{0}^{k}}{8 c_{N}}\right]^{\frac{2}{3+k}}, \\
& a_{c}=\left[\frac{\pi(1+k)^{2}(3+k)^{2} R^{2} \Delta \gamma z_{0}^{k}}{8 c_{N}}\right]^{\frac{1}{3+k}}, \\
& F_{c}=-\frac{3+k}{2} \pi \Delta \gamma R,
\end{aligned}
$$


and the generalized Tabor parameter for power-law elastic grading, i.e. the ratio of the characteristic height of the adhesive neck and the adhesion range:

$$
\Lambda:=\frac{\left|d_{c}\right|}{h},
$$

Equations (28) can be written in the form:

$$
\begin{gathered}
\tilde{d}=\frac{3+k}{1-k} \tilde{a}^{2}-\frac{16 \cos (k \pi / 2)}{\pi(1-k)^{3}} \Lambda \tilde{a}^{1-k} \sqrt{\left(m^{2}-1\right)^{1-k}} \\
\tilde{F}=\tilde{a}^{3+k}-\frac{2}{\pi} \frac{1+k}{1-k} \Lambda(m \tilde{a})^{2}\left[\pi+\frac{4 \cos (k \pi / 2)}{1-k^{2}} \frac{1}{m^{1+k}} .\right. \\
\cdot\left\{\sqrt{\left(1-\frac{1}{m^{2}}\right)^{1-k}}-{ }_{2} \mathrm{~F}_{1}\left(\frac{k-1}{2}, \frac{1+k}{2} ; \frac{3+k}{2} ; \frac{1}{m^{2}}\right)\right\} .
\end{gathered}
$$

The compatibility condition (29) in dimensionless variables reads:

$$
\begin{aligned}
& \frac{1}{\Lambda}=\tilde{d}\left[\frac{2}{\pi} \frac{\cos (k \pi / 2)}{1+k} \frac{1}{m^{1+k}}{ }_{2} \mathrm{~F}_{1}\left(\frac{1+k}{2}, \frac{1+k}{2} ; \frac{3+k}{2} ; \frac{1}{m^{2}}\right)-1\right]- \\
&-\frac{(m \tilde{a})^{2}}{2(1-k)}\left[\frac{4}{\pi} \cos \left(\frac{k \pi}{2}\right) \frac{1}{m^{3+k}}{ }_{2} \mathrm{~F}_{1}\left(\frac{1+k}{2}, \frac{3+k}{2} ; \frac{5+k}{2} ; \frac{1}{m^{2}}\right)-(1+k)(3+k)\right]- \\
&-\frac{32}{\pi^{2}} \frac{\cos ^{2}\left(\frac{k \pi}{2}\right) \Lambda(m \tilde{a})^{1-k}}{(1-k)^{3}(1+k)}\left[\frac{\Gamma(1-k) \Gamma\left(\frac{3+k}{2}\right)}{\Gamma\left(\frac{3-k}{2}\right)}-\frac{1}{m^{1+k}}{ }_{2} \mathrm{~F}_{1}\left(k, \frac{1+k}{2} ; \frac{3+k}{2} ; \frac{1}{m^{2}}\right)\right],
\end{aligned}
$$

which in the homogeneous case coincides with Maugis' solution [12] (Maugis uses a slightly different scaling for normalization). The JKR limit is given by the known relations [17]:

$$
\begin{aligned}
\tilde{d}^{\mathrm{IKR}} & =\frac{3+k}{1-k} \tilde{a}^{2}-\frac{4}{1-k} \tilde{a}^{\frac{1-k}{2}}, \\
\tilde{F}^{\mathrm{JKR}} & =\tilde{a}^{3+k}-2 \tilde{a}^{\frac{3+k}{2}} .
\end{aligned}
$$

As the adhesive force in the DMT limit,

$$
F_{\text {adh }}^{\text {DMT }}=2 \pi \Delta \gamma R,
$$

is independent of the elastic contact properties (it is actually the force for the adhesive contact of rigid spheres derived by Bradley [18]), the DMT limit of Eqs. (33) reads: 


$$
\begin{aligned}
\tilde{d}^{\mathrm{DMT}} & =\frac{3+k}{1-k} \tilde{a}^{2}, \\
\tilde{F}^{\mathrm{DMT}} & =\tilde{a}^{3+k}-\frac{4}{k+3} .
\end{aligned}
$$

To illustrate above findings and the influence of material grading Figs. 1 and 2 show the implicitly defined force-indentation relations as well as the respective JKR- and DMT limits for two different values of the power-law exponent $k$.

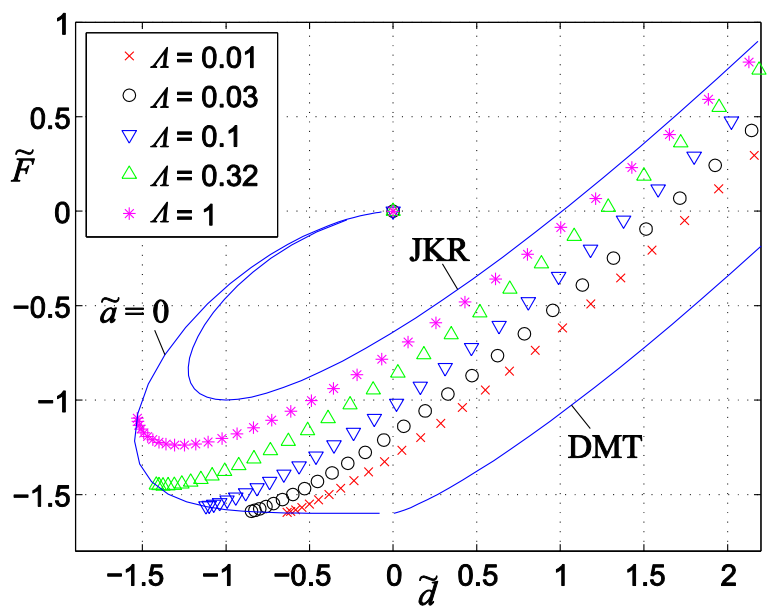

Fig. 1 Force-indentation-curves for the Dugdale-Maugis adhesive normal contact of powerlaw graded elastic spheres for $k=-0.5$ and several values of the Tabor parameter $\Lambda$

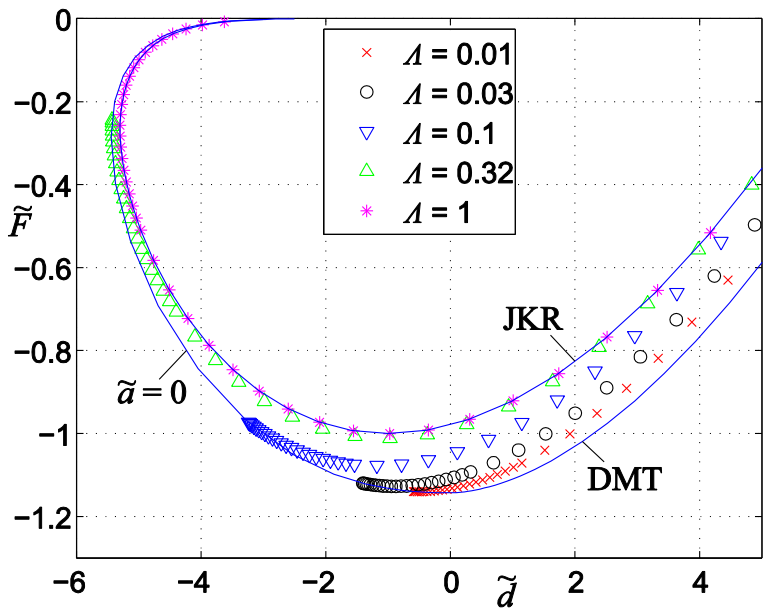

Fig. 2 Force-indentation-curves for the Dugdale-Maugis adhesive normal contact of powerlaw graded elastic spheres for $k=0.5$ and several values of the Tabor parameter $\Lambda$ 
Note that the DMT limit is only well-defined for positive indentation depths although the branch without direct contact (and therefore negative indentation depths) can be seen as its "natural" continuation. To denote this slight distinction a small gap is left between the DMT limit and the curve without direct contact in Fig. 1. Obviously the convergence for higher values of the Tabor parameter towards the JKR limit is much faster for larger values of $k$. For $k=0.5$ there is already no noticeable difference between the solution for $\Lambda=1$ and the JKR limit. Also the normalized indentation depths are getting much higher for larger values of $k$. Interestingly, the critical pull-off forces in the JKR- and DMT limit are the same for $k \rightarrow 1$ (as it was pointed out already in [6]). In this case the left branch of the JKR curve and the curve without direct contact will be practically indistinguishable.

\section{CONCLUSIONS}

Based on the MDR a closed-form analytic solution has been obtained for the DugdaleMaugis adhesive normal contact of arbitrary convex axisymmetric, power-law graded elastic bodies. As the most common and probably most relevant special case the contact of spherical or parabolic bodies has been studied in detail. The common limits for very large (JKR) or very small (DMT) values of the Tabor parameter are derived from the general solution. In dimensionless variables the relations between indentation depth, contact radii and normal force only depend on the Tabor parameter and exponent $k$ of the elastic grading. Thereby the convergence for larger values of the Tabor parameter towards the JKR limit is faster for higher values of $k$.

The presented solution is of course based on strong contact-mechanical assumptions (half-space hypothesis, absence of friction or roughness) and quantitatively problematic physical models (power-law grading with either infinitely stiff or infinitely soft surfaces, Dugdale model for the adhesive stress); it is, however, to the author's best knowledge, the only tool, to rigorously study the influence of both material grading and adhesion range in a closed form, for example in micro- or nano-applications, for which the range of the (adhesive) molecular forces becomes relevant. And although other models might seem physically more appropriate, they will probably neither allow for analytic treatment nor show a qualitatively different behavior.

For future work it would be interesting to compare the obtained analytical results with numerical or experimental findings.

\section{REFERENCES}

1. Suresh, S., 2001, Graded Materials for Resistance to Contact Deformation and Damage, Science, 292, pp. 2447-2451.

2. Scherge, M., Gorb, S., 2001, Biological Micro- and Nano-Tribology - Nature's Solutions, Springer, Berlin Heidelberg.

3. Booker, J.R., Balaam, N.P., Davis, E.H., 1985, The Behaviour of an Elastic Non-Homogeneous HalfSpace. Part I-Line and Point Loads, International Journal for Numerical and Analytical Methods in Geomechanics, 9(4), pp. 353-367.

4. Giannakopoulos, A.E., Suresh, S., 1997, Indentation of Solids with Gradients in Elastic Properties: Part I. Point Forces, International Journal of Solids and Structures, 34(19), pp. 2357-2392.

5. Giannakopoulos, A.E., Suresh, S., 1997, Indentation of Solids with Gradients in Elastic Properties: Part II. Axisymmetric Indentors, International Journal of Solids and Structures, 34(19), pp. 2393-2428. 
6. Chen, S., Yan, C., Zhang, P., Gao, H., 2009, Mechanics of adhesive contact on a power-law graded elastic half-space, Journal of the Mechanics and Physics of Solids, 57(9), pp. 1437-1448.

7. Guo, X., Jin, F., Gao, H., 2011, Mechanics of non-slipping adhesive contact on a power-law graded elastic half-space. International Journal of Solids and Structures, 48(18), pp. 2565-2575.

8. Jin, F., Guo, X., Zhang, W., 2013, A Unified Treatment of Axisymmetric Adhesive Contact on a PowerLaw Graded Elastic Half-Space. Journal of Applied Mechanics, 80(6), 061024.

9. Liu, Z., Meyers, M.A., Zhang, Z., Ritchie, R.O., 2017, Functional gradients and heterogeneities in biological materials: Design principles, functions, and bioinspired applications, Progress in Materials Science, 88, pp. 467-498.

10. Johnson, K.L., Kendall, K., Roberts, A.D., 1971, Surface Energy and the Contact of Elastic Solids. Proceedings of the Royal Society of London, Series A, 324, pp. 301-313.

11. Derjaguin, B.V., Muller, V.M., Toporov, Y.P., 1975, Effect of contact deformations on the adhesion of particle, Journal of Colloid and Interface Science, 53(2), pp. 314-326.

12. Maugis, D., 1992, Adhesion of spheres: The JKR-DMT-transition using a Dugdale model. Journal of Colloid and Interface Science, 150(1), pp. 243-269.

13. Dugdale, D.S., 1960, Yielding of steel sheets containing slits, Journal of the Mechanics and Physics of Solids, 8(2), pp. 100-104.

14. Tabor, D., 1977, Surface forces and surface interactions, Journal of Colloid and Interface Science, 58(1), pp. 2-13.

15. Heß, M., 2016, A simple method for solving adhesive and non-adhesive axisymmetric contact problems of elastically graded materials, International Journal of Engineering Science, 104, pp. 20-33.

16. Heß, M., Popov, V.L., 2016, Method of Dimensionality Reduction in Contact Mechanics and Friction: A User's Handbook. II. Power-Law Graded Materials, Facta Universitatis-Series Mechanical Engineering, 14(3), pp. 251-268.

17. Popov, V.L., Heß, M., Willert, E., 2018, Handbuch der Kontaktmechanik - Exakte Lösungen axialsymmetrischer Kontaktprobleme, Springer, Berlin Heidelberg.

18. Bradley, M.A., 1932, The cohesive force between solid surfaces and the surface energy of solids, The London, Edinburgh, and Dublin Philosophical Magazine and Journal of Science, 13(86), pp. 853-862. 